\title{
MAC Protocol for Wireless ATM - Channel Reservation Methods
}

\author{
Andrzej Stelter \\ Poznań University of Technology, Institute of Electronics \& Telecommunications \\ Email: astelter@et.put.poznan.pl
}

Key words: Wireless ATM, Medium Access Control

\begin{abstract}
The paper describes and evaluates by simulation an efficient channel access protocol (MAC protocol) based on TDMA scheme. Two methods of the buffer status transmission from mobile terminal to base station have been considered and compared. The first method combines a random access channel with piggybacking $(\mathrm{R}+\mathrm{PGBK})$, the second one combines a random access channel with backlog collision free slots assigned to the selected connections $(\mathrm{R}+\mathrm{B})$. The methods have been compared in two different systems. Simulation results show that for both proposed systems, $R+B$ method is better.
\end{abstract}

\section{INTRODUCTION}

In recent years, wireless ATM (WATM) has been a hot topic in telecommunications research. Typically WATM is considered as the wireless sub-domain of the B-ISDN infrastructure. Very important issue in such systems is MAC mechanism. As the RF bandwidth is a very limited resource, an effective channel utilization is extremely important for the wireless systems. In literature there are presented several medium access protocol propositions for WATM [2,3,4]. In majority of cases, ATM transmission is based on TDMA scheme, using TDD for the communication among the base station and the mobile terminals. Multiple access protocol is based on a TDMA time frame. Typically the protocol is centralized, i.e. it is controlled by the base station. The base station assigns the time slots (bandwidth) to the terminals which cooperate with it. To do the job 
efficiently, the base station must have sufficient knowledge concerning ATM cells waiting in the terminal output buffers. The terminal output buffer status information is transmitted by a short packet in a random access channel. The performance of such protocols depends on several factors [5]:

- uplink channel reservation method (the way in which mobile terminals reserve the uplink channel bandwidth),

- scheduling algorithm (algorithm used by the base station to assign time slots to individual terminals or connections),

- collision resolution algorithm (algorithm performed by terminals whose request packets have collided in a random access channel),

- the length of the frame.

In the paper only the first from the above mentioned four factors is studied. As has been stated, the terminals reserve the uplink channel bandwidth by sending short request packets in a random access channel. Because of possible collisions, such solution does not guarantee that the base station will have the packet on time. This would be particularly harmful for the real time connections. In majority of the WATM multiple access protocols there are implemented additional ways to transmit the buffer status packets in a collision free manner. The particular solutions presented in the literature are difficult to compare. The reason is lack of any common standard concerning simulated system assumptions.

In the simulation presented in this paper there are considered the protocols in which a random access channel is combined with one of two collision free transmission methods, either the output buffer status information is piggybacked onto ATM cell transmitted uplink [3,4], or it is sent in a minislot assigned to the selected connection [2].

\section{PROTOCOL DESCRIPTION}

In the paper it is considered a wireless network microcell with one base station and several buffered mobiles. There is studied a time-slotted duplex system in which the uplink (mobile-to-base) and downlink (base-to-mobile) communications are time multiplexed on a single frequency channel. The terminals do not communicate directly among themselves. Packets (ATM cells) waiting for transmission in each terminal are stored in the output buffers. Information concerning each buffer state is sent to the base station where it is used to assign a time slot to the connection.

In the considered time slotted system, one ATM cell is transmitted in one time slot. The slots are grouped in frames. The frame structure is shown in Figure 1. The frame starts with a header transmitted downlink by the base station. The header contains information about the frame structure and the 
acknowledgements for packets transmitted in the previous frame. The second field of the frame contains downlink slots D in which ATM cells are transmitted from base station to the mobile terminals. In the next field of the frame there are uplink slots U in which ATM cells are sent from terminals to the base station. Each packet transmitted in U and D slot comprises an ATM cell and two bytes of CRC code. In the frame, besides the uplink (U) and downlink (D) slots, there are short request slots $R$ in which terminals send a buffer status information, i.e. the number of cells in the buffer queue. The $R$ slots create slotted ALOHA type channel. When collision occurs in a slot $R$, the packet is retransmitted in the next frames according to the binary exponential backoff algorithm (probabilities of the packet retransmission in the subsequent frames diminish in the following way: $1 / 2,1 / 4,1 / 8, \ldots)$.

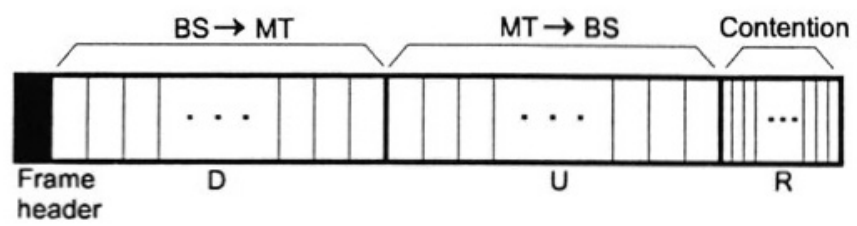

Figure 1. Frame structure

In the presented simulation, terminals are able to send the buffer status information not only in slots $\mathrm{R}$ but also

- piggybacking this information (PGBK field) onto transmitted uplink

ATM cells ( $\mathrm{R}+\mathrm{PGBK}$ method) or

- sending this information in additional slots $B$ assigned to the rt-VBR connections ( $\mathrm{R}+\mathrm{B}$ method).

Because ATM cells can be buffered in terminals in different ways, the two mentioned above channel reservation methods $(\mathrm{R}+\mathrm{B}$ and $\mathrm{R}+\mathrm{PGBK})$ are compared in two systems, which differ in terminal buffer organization (see Figure 2). Because of different buffer organization, the information transmitted in minislots B and R and in the field PGBK is different in both studied systems.

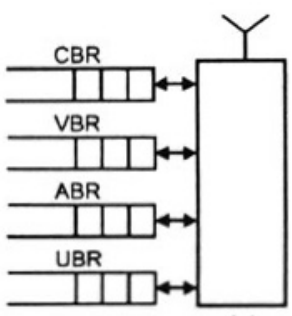

(a)

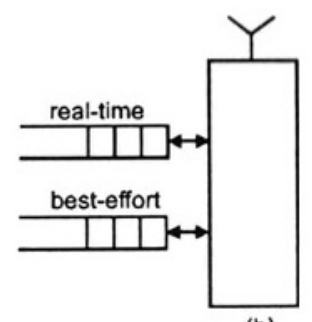

(b)

Figure 2. Terminal buffers: a) System I, b) System II 


\section{SYSTEM I}

In System I, each connection has its own identifier unique in the microcell (hence it is not necessary to identify terminals) and each connection has its own buffer in the terminal (see Figure 2a). Each ATM cell in a buffer is characterized by a 'delay', which rises with time. Cells in the buffers are arranged in order of delays and always a cell of the maximum delay is the first in a queue. CBR and rt-VBR cells for which the delay exceeds the specified limits are rejected. nrt-VBR, ABR and UBR cells of maximum delay are rejected when their buffers are overloaded.

Two methods of transmission of buffer status information to the base station are studied. In case of R+PGBK (see Figure $3 a$ ), a slot R is used only when simultaneously two conditions are fulfilled i.e. the buffer assigned to the connection is empty and a new ATM cell is generated. In this method the slot $\mathrm{R}$ packet consists of 10-bit connection identifier connection id (it allows 1024 connections per microcell) and 2-bit number $n_{-}$cells which contains information about the number of new cells in the queue (not registered in the base station). Two-bit number piggybacked onto ATM cell contains number of new ATM cells in the buffer.

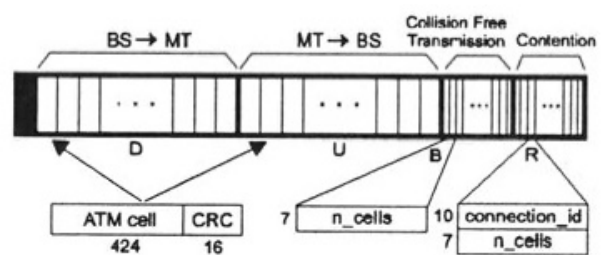

(a)

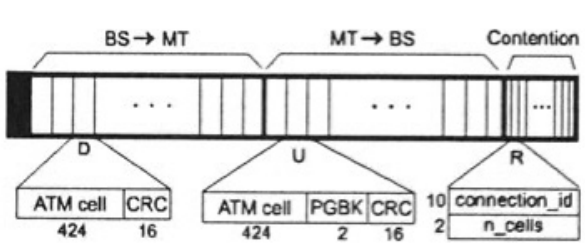

(b)

Figure 3. Channel reservation methods (System 1): a) $R+P G B K, b) R+B$

In case of R+B method (see Figure $3 b$ ), each rt-VBR connection has assigned a minislot $B$, hence transmission of the buffer status information of the $\mathrm{rt}-\mathrm{VBR}$ connection is collision free. Each rt-VBR connection has assigned a collision free slot B because rt-VBR traffic is characterized by a large range of changes and strict delay requirements. The seven-bit number $n$ cells in slots $\mathrm{R}$ and $\mathrm{B}$ is able to assign all frame slots to one connection when it works with maximum speed (it was assumed that the total number of transmission slots $\mathrm{D}$ and $\mathrm{U}$ in the frame is 100 ). In case of $\mathrm{R}+\mathrm{B}$ method the terminals having CBR, nrt-VBR, $A B R$ and $U B R$ connections have to compete for the channel access in slots $R$.

In the considered centralized system, the uplink slots $U$ in each frame are assigned to the connections by the base station. The base station handles a table, which contains a buffer descriptor for each connection. Each 
descriptor stores a number of ATM cells waiting for transmission in a buffer assigned to the connection. The table status is brought up to date after each successful packet transmission in the slot $R$ and the buffer status information taken from packet in slot $B(R+B$ method) or the buffer status information piggybacked on ATM packets in slots U (R+PGBK method).

\subsection{Simulation description}

The simulation results presented in the paper concern the uplink transmission. In the simulation all frames have constant length: $50 \times \mathrm{D}$ and $50 \times \mathrm{U}$ slots. It is assumed that the system transmission rate equals $20 \mathrm{Mb} / \mathrm{s}$, therefore one bit is transmitted in $50 \mathrm{~ns}$. It is assumed also that the radius of the wireless network microcell is equal to $45 \mathrm{~m}$, hence the maximum propagation delay equals $150 \mathrm{~ns}$ (3-bit interval). Each uplink slot is widened by this interval to avoid overlapping the signals transmitted by different terminals. Under the above assumptions the frame length equals $\sim 2.4 \mathrm{~ms}$. The uplink channel capacity equals $\sim 8 \mathrm{Mb} / \mathrm{s}$.

Two types of terminals that generate three traffic types (CBR, rt-VBR and $A B R$ ) are realized in the simulation. The first terminal is a videophone. It transmits both voice and video. The voice traffic represents CBR traffic (during voice activity), the video traffic represents real time VBR traffic. This type of terminal is denoted $T_{\mathrm{CBR}+\mathrm{VBR}}$. $\mathrm{CBR}$ traffic is modeled by a two-state Markov process. It is assumed that a mean length of the voice activity interval equals $1 \mathrm{~s}$, and a mean silence interval equals $1.35 \mathrm{~s}$. In active state CBR source generates a constant bit flow of the rate $32 \mathrm{~kb} / \mathrm{s}$. Maximum allowed delay of a cell generated by the above source equals 32 ms. CBR connection informs the base station (in a slot R) only about the first packet in a burst. After successful reception of the request packet, the base station assigns necessary slots periodically, with a constant rate.

rt-VBR traffic source was modeled using the method belonging to the class of discrete-time batch Markov arrival process [1]. For the rt-VBR source it is assumed that a mean bit rate is equal to $\mu=256 \mathrm{~kb} / \mathrm{s}$, standard deviation $\sigma=128 \mathrm{~kb} / \mathrm{s}$ and autocovariance $\mathrm{C}(\tau)=\sigma^{2} \mathrm{e}^{-\mathrm{a} \tau}\left(\mathrm{a}=3.9 \mathrm{~s}^{-1}\right)$. Values of the above parameters can describe a video source with no rapid movements in the scene. It is assumed that the maximum delay of ATM cells generated by the above rt-VBR source is $20 \mathrm{~ms}$.

The second terminal type, denoted $\mathrm{T}_{\mathrm{ABR}}$, realizes $\mathrm{ABR}$ packet data transmission. ABR traffic is modeled by the Poisson process. Each $T_{A B R}$ terminal generates data flow of the rate $256 \mathrm{~kb} / \mathrm{s}$. ABR buffer size is $100 \mathrm{~KB}$, hence for the mean rate of $256 \mathrm{~kb} / \mathrm{s}$ the maximum delay is $\sim 3 \mathrm{~s}$.

In the simulated system CBR connections have the highest priority, ABR connections have the lowest priority. This means that at first the base station 
assigns slots $\mathrm{U}$ to $\mathrm{CBR}$ connections, after that slots $\mathrm{U}$ are assigned to rt-VBR connections and next slots $U$ are assigned to $A B R$ connections.

All connections from terminals $\mathrm{T}_{\mathrm{CBR}+\mathrm{VBR}}$ and $\mathrm{T}_{\mathrm{ABR}}$ are established at the beginning of the simulation and are active during the whole simulation process. For a selected input traffic the number of terminals is constant during the simulation. The input traffic in the simulated system is defined by the number of $T_{C B R+V B R}$ and $T_{A B R}$ terminals. The simulation always covers $1200 \mathrm{~s}$ of the system activity for a given input traffic.

\subsection{Simulation results}

The simulation results presented in this chapter were previously published in [6]. The simulation was performed for three different traffic scenarios. The scenarios differ in contribution of CBR, rt-VBR and ABR traffic in the whole input traffic. In all three scenarios the contribution of CBR traffic in the whole input traffic is low. The majority of the input traffic is generated by rt-VBR and ABR connections. In the first scenario the traffic generated by the rt-VBR connections is the same as the traffic generated by the ABR connections. In the second scenario predominates rt-VBR traffic and in the third scenario predominates ABR traffic. The results obtained for all three scenarios are similar. For lack of space only results obtained for the first scenario are presented.

In the first scenario the number of $T_{C B R+V B R}$ terminals is the same as $T_{A B R}$ terminals. The contribution of $\mathrm{CBR}$, rt-VBR and $\mathrm{ABR}$ connections in the whole input traffic equals $2.6 \%, 48.7 \%$ and $48.7 \%$ respectively. The number of terminals of each type is changed from 8 to 34 , hence the input traffic changes from 4.2 to $17.8 \mathrm{Mb} / \mathrm{s}$.

For the $R+B$ protocol a number of slots $R$ equals 7 . This number ensures that $C B R$ cell loss ratio is less than 0.01 . For $R+P G B K$ protocol a number of the slots $R$ has to increase because they are utilized also by the rt-VBR connections. The simulation results are shown for this number equal 20, 40 and 60. CBR cell loss ratio for the above mentioned numbers is zero.

It is worth to mention that for the whole input traffic range and all protocol versions the average CBR cell delay equals to the length of the frame i.e. $\sim 2.4 \mathrm{~ms}$.

Figure 4 shows the average delay of rt-VBR ATM cells versus the number of terminals. The results show that the delay for $R+B$ method is less than for the R+PGBK method. The reason is that in $\mathrm{R}+\mathrm{B}$ method the base station is aware of the new rt-VBR ATM cells, waiting for transmission, with the delay at most equal to the length of the frame. In R+PGBK method, $\mathrm{rt}-\mathrm{VBR}$ connection informs the base station about its buffer status in a slot $\mathrm{R}$. Because of collisions the average delay is greater in this method. 


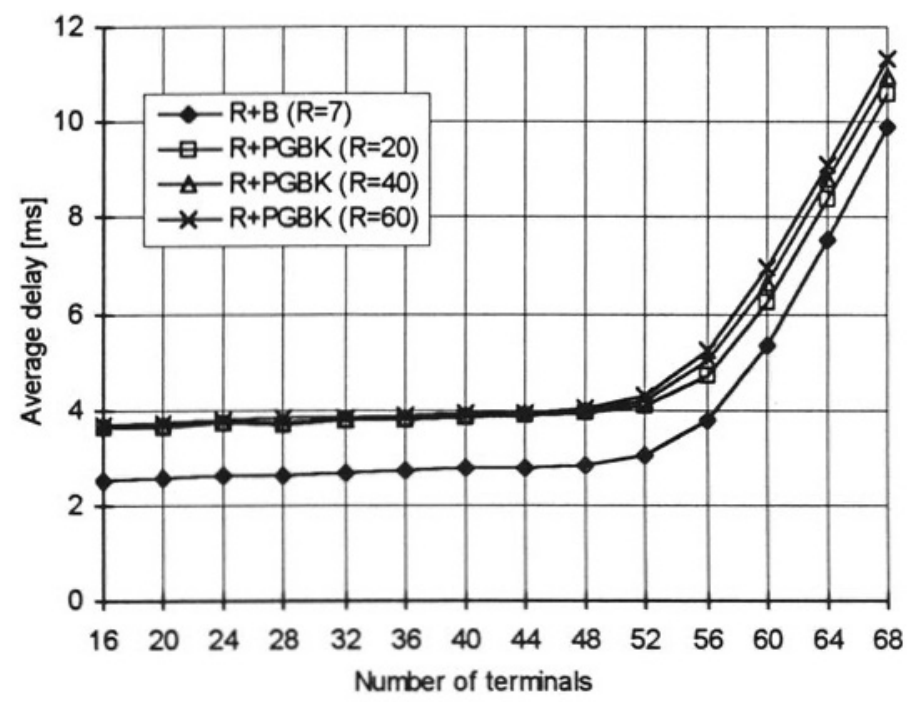

Figure 4. Average delay vs. input load for $n$-VBR connections (System I)

The average delay of ABR cells for both versions of the protocol ( $R+B$ and $\mathrm{R}+\mathrm{PGBK}$ ) is very similar. It is not presented in the paper.

Figure 5 shows the rt-VBR ATM cell loss ratio. During the simulation, when $T_{C B R+V B R}$ terminals generate input traffic less than the channel capacity, we do not observe any cell rejections for the $\mathrm{R}+\mathrm{B}$ protocol. A different result concerns $\mathrm{R}+\mathrm{PGBK}$ protocol. For this protocol, even for small input traffic, we observe some cell rejections. In this case the cell loss ratio depends on the number of the slots $\mathrm{R}$. We can notice that the cell loss ratio starts to decrease when the number of terminals increases and crosses the channel capacity (see Figure 5, 32 $\div 44$ terminals). This rather strange phenomenon can be explained by the fact that when there are not enough slots $\mathrm{U}$ in the system they are assigned mainly to rt-VBR connections because these connections have higher priority than ABR connections. The buffers for $\mathrm{ABR}$ connections are filling and $\mathrm{ABR}$ connections use slots $\mathrm{R}$ very seldom. Hence, even if in the system there are more terminals generating $A B R$ traffic, they use slots $R$ rarely and more of these slots can be used by the rt-VBR connections. With the increase of the input traffic (above 44 terminals) the cell loss ratio increases. From Figure 5 it can be noticed also that increasing the number of $\mathrm{R}$ slots, from 20 to 60 , decreases the cell loss ratio. The cost is the greater redundancy in the system.

Figure 6 shows the cell loss ratio for ABR connections. ABR cells are rejected when the number of terminals increases above 28 (above the channel capacity). The lowest cell loss ratio is observed for $\mathrm{R}+\mathrm{B}$ method 
because of the smallest redundancy in the system. In R+PGBK method the cell loss ratio increases with the number of the $\mathrm{R}$ slots because they diminish the system throughput.

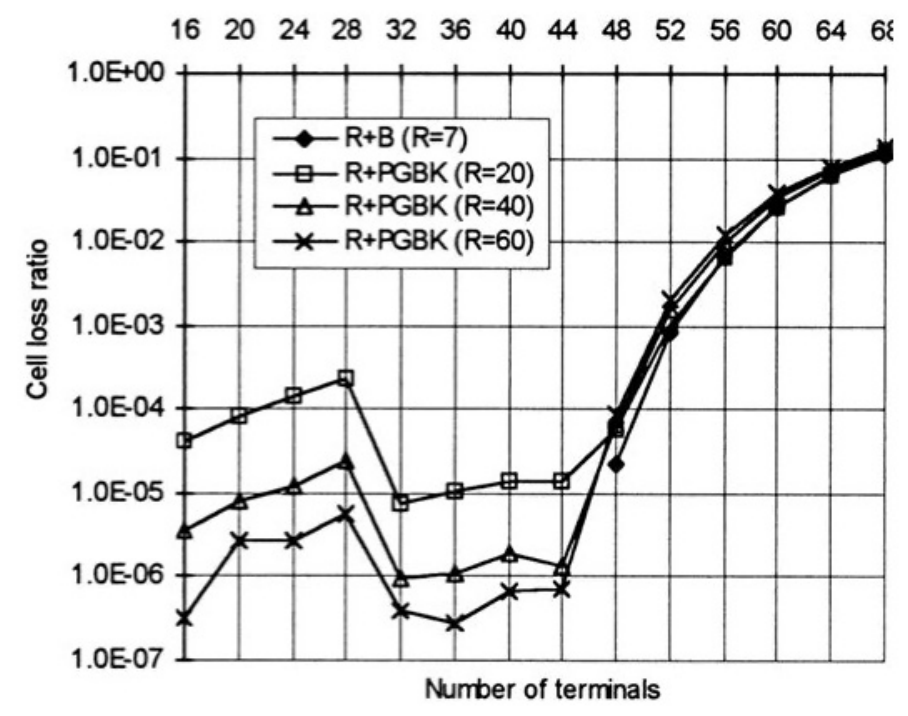

Figure 5. Cell loss ratio vs. input load for rt-VBR connections (System I)

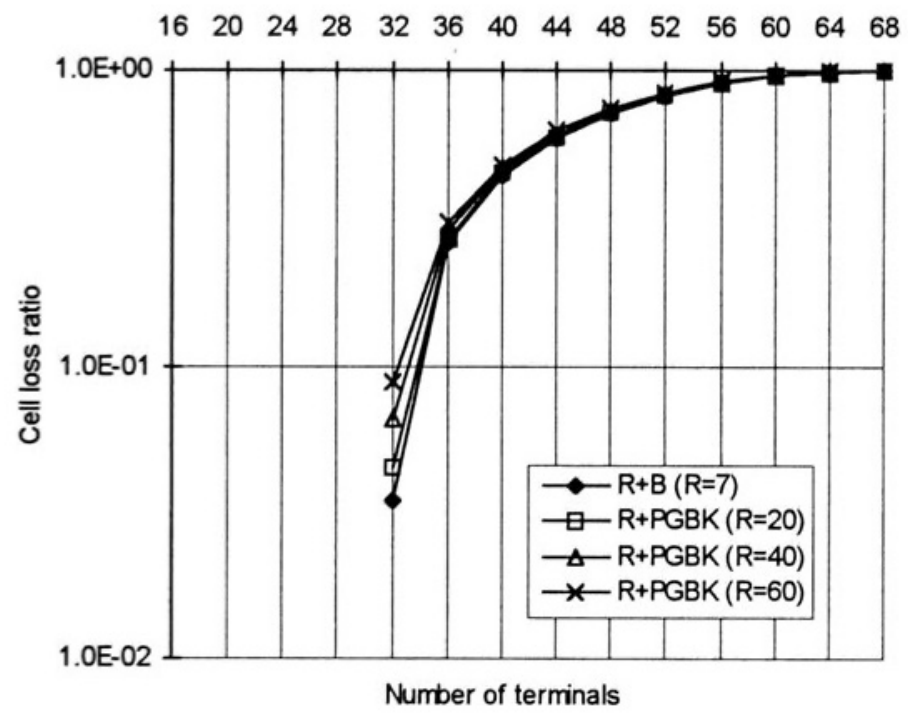

Figure 6. Cell loss ratio vs. input load for ABR connections (System I) 
Comparing results shown in Figure 5 and Figure 6 for a small traffic (number of terminals less than 32) it can be noticed that the cell loss ratio for ABR connections is better than for rt-VBR connections, even the last ones have higher priority. The reason is that the maximum delay for ABR cells is $\sim 3 \mathrm{~s}$ and the maximum delay for rt-VBR cells is $20 \mathrm{~ms}$. Therefore frequent collisions in the channel $R$ force very quickly the terminal realizing rt-VBR connection to reject the oldest cell in the buffer. The terminal realizing ABR connection has more time to inform the base station about its buffer status.

\section{SYSTEM II}

In System II, the packets (ATM cells) are buffered at the mobile terminals in two queues (Figure $2 b$ ). In the first queue (real-time queue) there are cells from all real-time connections (CBR, rt-VBR). In the second queue (best-effort queue) there are cells from other connections (nrt-VBR, ABR, UBR). Cells in both queues are arranged in order of deadlines. Cell of the shortest deadline is the first. There are two similar virtual queues in the base station. These queues keep references to remote packets, which are stored at mobile terminals waiting to be transmitted to the base station. When the base station assigns time slots $U$ to the mobiles, the real-time queue cells have high priority, the best-effort queue cells have low priority. The real-time queue cells are transmitted before the best-effort queue cells.

The same (as in System I) two methods of uplink channel reservation were evaluated by simulation in System II. The simulation parameters were similar to that used in the simulation of System I. The only difference were connection types realized in the system. There were assumed identical traffic characteristics for all mobiles. Each mobile terminal generates rt-VBR traffic and ABR traffic thus in each terminal two kinds of buffers (real-time and best effort) are created. Both rt-VBR and ABR sources were modelled by the methods described in the previous chapter. The simulation was performed for two different traffic scenarios. In the first scenario each terminal generates rt-VBR traffic of the mean rate equal to $256 \mathrm{~kb} / \mathrm{s}$ (maximum allowable delay $\tau=10 \mathrm{~ms}$ ) and ABR traffic of the average bit rate equal to $100 \mathrm{~kb} / \mathrm{s}(\tau=500 \mathrm{~ms})$. The number of mobiles varies from 14 to 40 , so the system input uplink traffic is equal to $\sim 5 \div 14.2 \mathrm{Mb} / \mathrm{s}$. In the second scenario each terminal generates $\mathrm{rt}-\mathrm{VBR}$ traffic of the mean rate equal to $2 \mathrm{Mb} / \mathrm{s}$ $(\tau=50 \mathrm{~ms})$ and ABR traffic of the average bit rate equal to $1 \mathrm{Mb} / \mathrm{s}(\tau=1 \mathrm{~s})$. The number of mobiles varies from 1 to 4 , the input traffic rate increases from $3 \mathrm{Mb} / \mathrm{s}$ to $12 \mathrm{Mb} / \mathrm{s}$. Results of the simulation of System II were published in [7]. They are similar to that obtained for System I. 


\section{CONCLUSION}

From the results of the simulation we see that for both systems considered in the paper, $\mathrm{R}+\mathrm{B}$ method to send buffer status information in the uplink channel is better than R+PGBK one. It gives higher system throughput, smaller average access delay and smaller cell loss ratio for all studied connection types. In R+PGBK method the rt-VBR cell loss ratio can be decreased by an increase of the number of $R$ slots, but it makes other parameters of the system (e.g. ABR connection parameters) worse because of higher redundancy in the system.

A disadvantage of $R+B$ method (in comparison with $R+P G B K$ ) can be the necessity for terminal having rt-VBR connection to turn on its transmitter one time more in every frame. This can cause larger power consumption.

Conclusions presented above concern a system with the error free wireless channel and perfect synchronization. That is why packets transmitted in $\mathrm{B}$ and $\mathrm{R}$ minislots are not protected and no synchronization fields are added to them. In practical system however errors will appear, thus packets in $B$ and $R$ minislots should be protected - some redundant bits should be added to these packets. In both methods ( $R+B$ and $R+P G B K)$ the number of minislots ( $B$ and/or $R$ ) does not differ significantly, so it suggests that not taking into consideration redundant bits in the minislots has little influence on the result of comparison of both studied methods. This problem is studied by the author in detail at present.

In practical system all packets should be extended by a header containing a synchronization pattern. That will decrease the system throughput but should not influence the result of comparison of $\mathrm{R}+\mathrm{B}$ and $\mathrm{R}+\mathrm{PGBK}$ method.

\section{REFERENCES}

[1] C. Blondia, O. Casals, "Performance Analysis of Statistical Multiplexing of VBR Sources", Proceedings of INFOCOM'92, pp. 828-838, 1992

[2] N. R. Figueira, J. Pasquale, "Remote-Queueing Multiple Access (RQMA): Providing Quality of Service for Wireless Communications", Proceedings of INFOCOM'98, pp. 307-314, 1998

[3] N. Passas, S. Paskalis, D. Vali, L. Merakos, "Quality-of-Service-Oriented Medium Access Control for Wireless ATM Networks", IEEE Comm. Magazine, pp. 42-50, Nov. 1997

[4] D. Petras, "Medium Access Control Protocol for Wireless Transparent ATM Access", IEEE Wireless Comm. Systems Symposium, Long Island NY, pp. 79-84, Nov. 1995

[5]A. Stelter, "Medium Access Control Protocols for Wireless ATM System", Poznan University of Technology, $\mathrm{PhD}$ thesis, Jan. 2000 (in Polish)

[6] A. Stelter, P. Szulakiewicz, "ATM MAC Protocol for the Radio Interface - Performance Evaluation", Proceedings of PIMRC'99, Osaka, Japan, 1999

[7] A. Stelter, P. Szulakiewicz, "Wireless ATM MAC Protocol - Performance Evaluation by Simulation", Proceedings of WIRELESS 99, pp. 449-455, Calgary, Canada, 1999 